\title{
TTR
}

Traduction, terminologie, re?daction

\section{Le sujet en question : I am Yours de Judith Thompson, version québécoise}

\section{Louise Ladouceur}

Volume 11, numéro 1, 1er semestre 1998

Diachronie et synchronie

Diachronics and Synchronics

URI : https://id.erudit.org/iderudit/037317ar

DOI : https://doi.org/10.7202/037317ar

Aller au sommaire du numéro

Éditeur(s)

Association canadienne de traductologie

ISSN

0835-8443 (imprimé)

1708-2188 (numérique)

Découvrir la revue

Citer cet article

Ladouceur, L. (1998). Le sujet en question : I am Yours de Judith Thompson, version québécoise. TTR, 11(1), 89-112. https://doi.org/10.7202/037317ar
Résumé de l'article

Le Sujet en question : I Am Yours de Judith Thompson, version québécoise - Si certains choix de traduction sont orientés par les normes collectives en vigueur dans le contexte récepteur, il en est d'autres qui relèvent de l'espace plus restreint du champ dans lequel s'inscrivent l'activité de traduction et l'agent qui la pratique. D'autres encore procèdent des subjectivités participant à la production de l'oeuvre en traduction. Cet article traite des relations qu'entretient le sujet traduisant avec ces nombreuses contraintes dans Je suis à toi, version québécoise de la pièce I Am Yours de l'auteure canadienne-anglaise Judith Thompson.
Tous droits réservés (C) TTR: traduction, terminologie, rédaction — Les auteurs, 1998
Cedocument est protégé par la loi sur le droit d'auteur. L'utilisation des services d'Érudit (y compris la reproduction) est assujettie à sa politique d'utilisation que vous pouvez consulter en ligne.

https://apropos.erudit.org/fr/usagers/politique-dutilisation/ 


\section{Le sujet en question : I am Yours de Judith Thompson, version québécoise ${ }^{1}$}

\section{Louise Ladouceur}

La nature même de l'œuvre thétrale fait obstacle à la reconstitution du trajet suivi par le texte en traduction. Il y a d'abord le fait que le texte dramatique est rarement transmis exactement comme il est écrit puisqu'il est confié à plusieurs intermédiaires qui en font nécessairement une lecture subjective. Ainsi, le producteur posera des conditions qui auront un effet sur le texte, le metteur en scène exigera qu'on l'adapte à sa vision scénique et l'acteur le modulera selon son propre idiolecte ou l'enrobera de connotations qui en modifient la portée, voire le sens. Soumis aux nécessités de la représentation, le texte de théâtre participe en outre à un réseau complexe de codes sémiotiques avec lesquels il entretient d'étroites relations qui modèlent le message verbal livré sur scène. Tadeusz Kowzan définit cinq catégories de signes contribuant à l'acte thếtral : le texte dit, l'expression corporelle, l'apparence externe de l'acteur, l'espace de la performance et les sons non dits (Kowzan, 1975, pp. 52-80). La

${ }^{1}$ Cette étude est issue d'une recherche doctorale intitulé * Separate Stages : la traduction du théatre dans le contexte Canada/Québec ", laquelie a été subventionnte par le Conseil de recherches en sciences humaines du Canada. 
superposition de ces divers systèmes sémiotiques propres au spectacle fait en sorte que le texte de thêâtre " ne se livre que dans une épaisseur de signes synchroniques, c'est-à-dire étagés dans l'espace, spatialisés n (Ubersfeld, 1978, p. 153).

Dans le cas qui nous intéresse, il faut signaler aussi que la version traduite n'a pas été publiée, qu'elle existe sous forme manuscrite dactylographiée, dont un exemplaire a été déposé à la bibliothèque de l'École nationale de théâtre du Canada à Montréal. Il se pourrait que des changements effectués au cours des répétitions n'y figurent pas. Pour ajouter à l'énigme, la comparaison des textes d'arrivée et de départ se heurte au fait qu'on omet de mentionner l'approche privilégiée par le traducteur ainsi que la ou les versions originales dont on s'est inspiré. Bien que cette dernière omission soit courante dans le domaine de la traduction, elle prend, dans le cas du texte de théâtre, une valeur particulière. Contrairement aux autres produits littéraires, dont la publication fige d'emblée la forme, le texte dramatique peut circuler en versions manuscrites, remaniées au fil des productions et parfois fort dissemblables les unes des autres, avant d'être publié. Ce flottement donne lieu à une pratique très courante en traduction théâtrale : le rapiéçage textuel consistant à assembler des passages empruntés à différentes versions du texte original. Pour ce qui est de I Am Yours de la dramaturge canadienne Judith Thompson, il faut préciser que la pièce a été créé en 1987 mais n'a été publiée qu'en 1989, ce qui signifie que des versions manuscrites ont circulé entre-temps. Une lecture de la version manuscrite datant de $1987^{2}$ révèle que certains passages ont été considérablement modifiés pour la publication du texte en 1989. Bien que la version québécoise emprunte en majeure partie au texte de 1987 , certains passages ne peuvent résulter que du recours à d'autres versions antérieures à la publication ou d'une récriture lors de la traduction. Le manuscrit de 1987 et la version publiée de 1989 seront consultés pour l'étude qui suit.

${ }^{2}$ Cette version a été gracieusement fournie par Shain Jaffe de Great North Management, agent de Judith Thompson. 


\section{Invisible sujet}

Selon l'hypothèse exprimée par Gideon Toury dans les recueils $n$ Search of a Theory of Translation (1980) et Descriptive Translation Studies and Beyond (1995), c'est la fonction assignée au texte traduit dans le contexte récepteur qui oriente le choix des stratégies appliquées à sa traduction. Dans cette optique, la traduction littéraire est le produit de normes agissant sur le texte, d'abord au niveau linguistique, selon le système matriciel de la langue-cible, et ensuite au niveau littéraire, selon le modèle imposé par le système littéraire récepteur (Toury, 1980, pp. 36-37).

Elargie par Annie Brisset dans Sociocritique de la traduction. Théâtre et altérité au Québec (1968-1988) (1990), la perspective fonctionnaliste a par la suite dépassé le cadre du polysystème littéraire pour s'étendre au contexte socio-culturel qui l'englobe. S'inspirant de la réflexion sur le discours développée par Michel Foucault et du concept de « discours social " proposé par Marc Angenot ${ }^{3}$, Annie Brisset soutient que la traduction littéraire est façonnée non seulement par des contraintes linguistiques et littéraires, mais aussi par * l'ensemble des codes qui régissent à des degrés divers le discours de la société-cible " (Brisset, 1990 , p. 26). Ce cadre theorique lui a permis de faire voir l'ethnocentrisme à l'cuvre dans les imitations, parodies et traductions étudiées, lesquelles ont pour objet non pas de révéler l'œuvre empruntée mais de * prêter à cette cuvre étrangère le dessein de mettre en scène le "fait québécois" " (Brisset, 1990, p. 312).

La vertu première du modèle fonctionnaliste est de situer l'étude de la traduction littéraire dans le champ de l'institution et du contexte social dont elle procède, en mettant au premier plan les normes collectives auxquelles sont soumis les textes éudiés. Révélateur d'idées et de valeurs partagées par une collectivité donnée, le modẻle fonctionnaliste propose toutefois une vision homogène de cette collectivité en ce sens qu'il donne à voir ce qui y constitue la norme. Orienté vers l'étude du traduit dans sa dimension la plus englobante, le modèle ne peut rendre compte de

\footnotetext{
${ }^{3}$ Marc Angenot définit ainsi le discours social : * l'ensemble de ce qui se dit et s'écrit dans un état de société, dans la mesure où cet ensemble n'apparaît pas comme composé d'énoncés aléatoires, mais au contraire, réglés par des conventions, pris dans des configurations idéologiques $x(1979$, p. 63).
} 
contraintes agissant de façons plus particulières sur l'cuvre en traduction. S'il permet d'identifier certaines règles appliquées systématiquement au répertoire traduit à une époque donnée de l'évolution d'une littérature, il néglige toutefois les phénomènes traductifs qui n'obéissent pas à cette systématisation. Ainsi, il ne peut prendre en considération, entre autres, la position occupée par le sujet traduisant au sein de la collectivité et les dimensions subjectives du traduiret. Orientés par l'ensemble des normes gouvernant la production textuelle dans la société visée, les choix de traduction sont également régis par les rapports conscients et inconscients qu'entretient le sujet traduisant avec le texte, dans ses dimensions collective et individuelle. Comme le souligne Antoine Berman dans l'ouvrage intitulé Pour une critique des traductions : John Donne, une traduction est nécessairement individuelle puisqu'en demière instance elle " procède d'une individualité, même soumise à des "normes" "(Berman, 1995, p. 60).

Dans cet esprit, on pourrait se demander, par exemple, en quoi les innombrables choix effectués lors de la traduction sont déterminés par l'origine du traducteur ou de la traductrice, par son appartenance à un sexe et à une classe sociale. En quoi ces choix sont-ils orientés par l'éducation, l'expérience personnelle, l'orientation sexuelle et les allégeances politiques, par les convictions morales ou religieuses, les préférences esthétiques, bref, tout ce qui informe le sujet traduisant et le positionne au sein de la collectivité dont il fait partie? On pourrait aussi considérer l'influence d'autres individus intervenant dans la traduction à différentes étapes de sá réalisation, soit au niveau du jeu, de la mise en scène, de la production ou de la publication. Ainsi se pose la question des contraintes exercées sur les choix de traduction non seulement par le contexte social, son appareil littéraire et ses codes linguistiques, mais par le sujet traduisant soumis aux effets de sa propre subjectivité et de celle de ses collaborateurs.

\footnotetext{
${ }^{4}$ Luise von Flotow commente ainsi la " positionnalite " du sujet traduisant : " Though such subjective aspects of translation as the translator's biographical, psychological, ideological and professionnal background - her positionality are rarely reflected upon, they certainly have an impact on the final text " (1995, p. 32).
} 
Selon Berman, tout traducteur élabore une position traductive qui lui est propre et par laquelle il se constitue en tant que sujet :

La position traductive est, pour ainsi dire, le " compromis * entre la manière dont le traducteur perçoit en tant que sujet pris par la pulsion de traduire, la tâche de la traduction, et la manière dont il a * internalisé $*$ le discours ambiant sur le traduire (les * normes $*$ ). (Berman, 1995, p. 74-75)

La position traductive peut être reconstituée à partir des traductions ellesmêmes, qui les expriment implicitenent, et à partir des commentaires dont le traducteur accompagne sa pratique. Toutefois, Berman note que :

[...] ces représentations n'expriment pas toujours la vérité de la position traductive, notamment lorsqu'elles apparaissent dans des textes fortement codés comme les préfaces, ou des prises de parole conventionnelles comme les entretiens. Le traducteur, ici, a tendance à laisser parler en lui la doxa ambiante et les topoi impersonnels sur la traduction. (Berman, 1995, p. 75)

Cette méfiance envers la représentativité des propos que tient le traducteur sur sa position traductive n'est pas sans rappeler la mise en garde exprimée par Toury au sujet de la valeur réelle des normes puisées aux sources extratextuelles, que ce soit dans les commentaires, présentations ou préfaces portant sur la traduction, puisque celles-ci ont le plus souvent pour objectif de promouvoir plutôt que de révéler une façon de traduire. Cette réserve commune met en relief le caractère élusif d'un sujet traduisant dont les représentations auraient tendance à voiler plus qu'à révéler l'action. Serait-ce qu'il ne sait plus comment échapper à l'anonymat auquel il a été si longtemps confiné? L'invisibilité du travail effectué par la traduction, dont le mérite a longtemps consisté à passer inaperçu, est d'ailleurs vivement questionnée et problématisée par la critique traductologique récente. Dans le cadre de la réflexion féministe, par exemple, on a insisté sur la manifestation du sujet féminin traduisant qui affirme sa présence dans le texte, comme en font foi les traductions et 
les commentaires de Barbara Godard et de Susanne de LotbinièreHarwoods.

Selon Lawrence Venuti, l'impératif de transparence longtemps imposé à la traduction et voulant que cette dernière donne l'illusion d'être un original est lourd de conséquences :

What is so remarkable here is that this illusory effect [of transparency] conceals the numerous conditions under which the translation is made, starting with the translator's crucial intervention in the foreign text. (Venuti, 1995, p. 1)

On parle rarement, en effet, dans le discours critique portant sur la traduction des conditions matérielles dans lesquelles elle s'effectue, des allégeances dont elle procède, des relations de travail qu'elle met en jeu, des intérêts dont elle est l'enjeu. Entendons ici par " intérêts » ce que Pierre Bourdieu définit comme " investissement spécifique dans les enjeux, qui est à la fois la condition et le produit de l'appartenance à un champ * (Bourdieu, 1980, p. 119), et qui fait qu'on lutte pour maintenir ou améliorer sa position en tant qu'agent appartenant à un champ social déterminé. C'est en s'inspirant de la sociologie bourdieusienne que JeanMarc Gouanvic soulève la question suivante au sujet du traducteur : " dans quelle dynamique sociale s'inscrit-il dans le champ, autrement dit comment négocie-t-il sa position par rapport aux autres agents avec lesquels il est en concurrence pour la légitimité? " (1997, p. 37). Dans cette perspective, on peut concevoir que la traduction soit aussi tributaire des intérêts spécifiques du traducteur au sein du champ où il œuvre et du groupe auquel il appartient, qu'elle soit marquée par les rapports d'affinité et de concurrence des agents qui contribuent à sa production.

${ }^{5}$ Voir son livre Re-Belle et Infidèle. La traduction comme pratique de réécriture au féminin/The Body Bilingual. Translation as a Rewriting in the Feminine. Montréal/Toronto, Les Éditions du remue-ménage/Women's Press, 1991.

${ }^{6}$ L'application de la theorie bourdieusienne à la traductologie semble très prometteuse dans l'élaboration d'une véritable sociologie de la traduction. Voir, à cet effet, les travaux de Jean-Marc Gouanvic, plus particulièrement l'article * La traduction et le devenir social : le cas de l'irruption de la science-fiction américaine en France après la Seconde Guerre mondiale * (1994). 
Parce que la traduction est une activité le plus souvent invisible, elle est un site privilégié de manipulations de toutes sortes qui relèvent de la relation qu'entretient le sujet traduisant avec des contraintes d'ordres divers. Celles-ci peuvent émaner de l'espace plus large du contexte social, littéraire et linguistique; de celui, plus restreint, du champ spécifique dans lequel s'inscrit l'activité de traduction et de la position occupée par l'agent qui la pratique; et, enfin, du territoire plus intime où s'exprime la subjectivité des individus participant a la production de l'ouvre en traduction. C'est la manifestation de ces contraintes et de leurs rapports que nous tenterons de dégager dans l'étude des déplacements opérés dans la version québécoise de la pièce de Judith Thompson I Am Yours.

\section{Réception}

Cette pièce donne à voir la bataille féroce qu'on se livre pour un enfant qui n'est pas encore né. Incapable de recourir à l'avortement, Dee, une artiste de classe moyenne au tempérament instable et récemment séparée de son mari, projette de donner en adoption l'enfant issu d'une brève rencontre avec le concierge, Toilane, un rustaud aux idées bien arrêtées à qui elle refuse de donner l'enfant. Décidé à faire prévaloir ses droits paternels et encouragé par sa mère Pegs, une femme énergique à qui rien ne résiste, Toilane parviendra à kidnapper le nouveau-né juste avant que Dee avoue vouloir garder l'enfant.

Avec cette troisième pièce, créée au Tarragon Theatre en 1987, Judith Thompson remportait le Chalmers Canadian Play Award la même année à Toronto. Le texte fut publié en 1989, avec trois autres pièces de Thompson, The Crackwalker, Pink et Tornado, dans l'anthologie The Other Side of the Dark, pour laquelle l'auteure remportait un deuxième Prix du Gouverneur général et s'affirmait comme dramaturge canadienne de premier plan.

Décidément flamboyante, l'écriture de Thompson est ainsi décrite par la critique : * straight from the gut [...] discursive rather than linear " (Crew, 1987, p. B1), " an hallucinatory series of lightning flashes n (Bemrose, 1987, p. 65), $\propto$ an expression of passion and obsession in violent, unpredictable irrational modes " (Garebian, 1988, p. 232). C'est cette originalité qui aura sans doute attiré l'attention des producteurs québécois, lesquels étaient auparavant, selon un article paru dans The 
Globe and Mail en janvier 1993, * bored to tears [with] English Canada's love affair with a plodding American-style naturalism " (Conlogue, 1993, p. A12). Il faut dire que la ferveur nationaliste des années soixante-dix et l'apathie post-référendaire des années quatre-vingt n'auront pas aidé la cause, comme le suggère un bref commentaire tiré du même article : « It was more political before " (Conlogue, 1993, p. A12). Quoi qu'il en soit, alors que seulement sept pièces anglo-canadiennes ont été traduites au Québec avant 1980 et vingt autres de 1980 à 1989, un nombre record de dix pièces sont produites entre 1990 et 1993, avec I Am Yours de Judith Thompson ouvrant la marche, suivie de pièces de Brad Fraser, Hillar Litoja, Norm Foster, Peter Madden, Wendy Lill, Sally Clark et Tomson Highway.

Selon Pierre Bernard, directeur artistique du Théâtre de Quat'Sous, où seront produites en traduction une autre pièce de Thompson, Lion in the Streets (1991), et trois pièces de Brad Fraser, cet intérêt soudain pour la dramaturgie canadienne-anglaise tiendrait au fait que * the quality of writing is now stronger than before " (Conlogue, 1993, p. Al2). En ce qui a trait à l'auteure de I Am Yours, Bernard ajoute : " translated into French [...] her phantasmagoric urban landscapes are simply part of a modern world which could as easily be Montreal * (Conlogue, 1993, p. A12). C'est aussi l'avis de Fernand Rainville, qui a dirigé trois productions de pièces canadiennes-anglaises en traduction présentées en 1993 : « Her plays are very urban, very much Toronto. But what comes across in French is the feeling of the big city anywhere, as it is now : immigrants in the streets, tension, misunderstanding. It's pertinent " (Conlogue, 1993, p. A12). Cette universalité serait d'autant plus éloquente qu'elle s'énoncerait d'une façon bien particulière, comme le souligne Claude Poissant, qui a mis en scène les versions québécoises des deux pièces de Thompson : « I'm surprised she's English... Canadian English... It's like she wrote our energy... this excess, this energy, it's more latin, it's Italian, it's more like Quebec * (Nunn, 1991, p. 17). Ainsi, l'attrait exercé par les pièces de Thompson résiderait moins dans leur spécificité canadienne-anglaise que dans leur capacité d'exprimer une réalité universelle sur un mode québécois.

Inaugurant l'arrivée en force du théâtre canadien-anglais sur les scènes montréalaises, Je suis à toi, version québécoise de I Am Yours, est présentée en novembre 1990 au restaurant-thêâtre La Licome par le 
Théâtre de la Manufacture, troupe reconnue déjà pour l'audace de certaines productions. La mise en scène est de Claude Poissant, dont la réputation n'est plus à faire au sein des troupes se consacrant au thêatre de création, et la traduction est de Robert Vézina. Peu connu à l'époque, ce dernier traduira par la suite Lion dans les rues de la même auteure, aussi mis en scène par Claude Poissant au Theâtre de Quat'Sous en 1991, et Poor Super Man de Brad Fraser, mis en scène par Fernand Rainville au Quat'Sous encore, en 1995, et dans lequel Claude Poissant tenait le rôle titre. Il faut rappeler que Fernand Rainville a signé la mise en scène de deux autres pièces canadiennes-anglaises présentées en version québécoise en 1993. On peut donc voir se constituer ici un réseau d'individus travaillant en étroite collaboration dans le champ de la production québécoise du théâtre anglo-canadien en traduction.

L'accueil réservé par la presse à la production de Je suis à toi est loin d'être chaleureux. Un rare commentaire enthousiaste émerge parmi les comptes rendus platement anecdotiques et les critiques virulentes envers une écriture qui « n'a pas le génie de Tremblay »(Beaunoyer, 1990, p. D8) et a n'approfondit pas assez la psychologie des personnages" (Boulanger, 1990, p. 28) dans ce " chorus of grotesques" (Charlebois, 1990, p. 25) soumis au " plat déterminisme de Thompson "(Letourneur, 1991, p. 172). On s'en prend aussi à une mise en scène où $\alpha$ les antagonismes de classe[s] ont été gommés " (Letourneur, 1991, p. 172) et à une version québécoise "clumsily translated "(Charlebois, 1990, p. 25) où " tous les personnages ont été gratifiés du même parler populaire " (Letourneur, 1991, p. 172). Ce choix surprend en effet puisque la pièce originale insiste sur l'écart dans les niveaux de langue des personnages, lesquels appartiennent à deux classes sociales qui s'affrontent pour décider du sort de l'enfant à venir. L'emploi de ce procédé ne sera d'ailleurs pas sans conséquence sur le discours véhiculé par la traduction, comme nous le verrons plus loin dans cette étude. Enfin, les critiques apprécient * les formidables numéros d'acteur * (Boulanger, 1990, p. 28) qui n'arrivent cependant pas à sauver la production. Ainsi donc, avec cette première incursion dans le monde théatral franco-québecois, Judith Thompson n'aura pas reçu l'accueil escompté après le succès de la pièce originale.

Cependant, ce qui étonne davantage dans la réaction de la presse québécoise, c'est l'absence de commentaire sur l'impact que la pièce pouvait avoir dans une province qu'une violente polémique avait déchirée, 
deux ans plus tôt, lorsque Jean-Guy Tremblay eut recours à des mesures extrêmes pour empécher l'avortement projeté par sa conjointe Chantal Daigle. Cette odyssée judiciaire commença le 7 juillet 1989 quand, à la demande de Tremblay, la Cour supérieure du Québec émit une injonction interdisant à Daigle de procéder à l'avortement. La décision fut maintenue par la Cour d'appel le 26 juillet pour être ensuite renversée le 8 aout par la Cour suprême du Canada après qu'on l'eut informée que l'avortement avait eu lieu illégalement. L'affaire fut passionnément débattue dans les médias à travers tout le Québec et lors de nombreuses manifestations provie et pro-choix, dont une marche de protestation où plus de sept mille personnes défilèrent dans les rues de Montréal sous d'occasionnelles insultes jaillies des balcons avoisinants.

Comment, dans un tel contexte, la critique a-t-elle pu ignorer la résonance particulière d'une pièce où un père s'empare d'un nouveau-né que sa mère ne désire pas, bien qu'elle n'ait pu se résoudre à l'avortement? ${ }^{7}$ Plus étonnant encore, pourquoi les producteurs québécois ont-ils choisi de ne pas en parler? À cet effet, d'ailleurs, le programme accompagnant la production est empreint d'un vague lyrisme qui non seulement ne révèle rien du propos de la pièce, mais propose en outre un discours qui n'a rien de neutre. Voici un extrait du programme, où figure un bébé à la moue affligée sur le point d'éclater en sanglots : " Auronsnous le courage d'ouvrir nos yeux pour voir, nos oreilles pour entendre, et nos cceurs pour comprendre et répondre à ces bras tendus en tendant les nôtres? " (Leduc et Simard, 1990). Comment ne pas voir le discret message véhiculé dans ce prélude à une pièce traitant d'une grossesse non désirée?

\section{Déplacements}

Le recours à certains procédés de traduction suggère aussi un net parti pris dans le conflit central de la pièce. Par exemple, on a conservé dans la version québécoise les noms des personnages de l'œuvre originale, dont celui du père de l'enfant: Toi, diminutif de Toilane. Dans une pièce où les

\footnotetext{
${ }^{7}$ Seule Pat Donnelly du journal The Gazette fait allusion à la chose. Elle écrit : $\alpha$ In the province where Jean-Guy Tremblay went to legal extremes in an attempt to stop Chantal Daigle from having an abortion, Je Suis à Toi takes on a special resonance $*(1990, \mathrm{p} . \mathrm{D} 13)$.
} 
parents s'affrontent pour la possession de l'enfant, cette extrême fidélité onomastique donne au titre québécois $J e$ suis à toi une valeur que ne suggère nullement le titre original $I$ Am Yours. Il est difficile de ne pas voir les implications de ce jeu de mots, de prime abord astucieux, mais affichant néanmoins une prise de position sans équivoque. Je suis à toi ne peut qu'affirmer l'appartenance au père.

Un autre choix de traduction qui intervient dans la dynamique du conflit parental est l'attribution du même sociolecte populaire à tous les personnages de la pièce. Ce procédé a pour résultat d'occulter une fonction importante du langage, à laquelle Pegs fait d'ailleurs allusion en avouant ainsi son mépris envers la classe sociale représentée par Dee, son mari Mack et sa sœur Mercy :

PEGS. One day, I'm talkin to her and she up and corrects my grammar. Well, I turn around and says 'You think I don't know the correct grammar? I know it's "don't have any" but I say "don't got none". I CHOOSE "don't got none". I CHOOSE my grammar, cause I'd rather be dead; I'd rather be dead than be anything like you.' THEY HAVE US BELIEVIN WE CAN'T TALK, WE CAN'T DRESS, AND NOW THEY HAVE YOU BELIEVIN YOU DONT HAVE A RIGHT TO YOUR CHILD! (Thompson, 1987, p. 47; 1989, p. 160)

Voici un échange entre Pegs, Dee et Mack dans lequel on peut observer les marques distinctes du niveau de langue attribué à Pegs (lesquelles sont mises en italiques) :

MACK. Come on! We'll all sit down!

DEE. If you don't leave I'm calling the police.

MACK. Oh my God there's no need to bring the police into this my dear, let's listen to what the woman has to say.

DEE. Well, I'm leaving then, you can all stay here.

MACK. Dee, come on, relax.

PEGS. Enough of this stupidity. YOU are gonna give my grandchild away over my DEAD BODY. (terrible pause) You hear me?

DEE. I'm sorry, I really don't know what you're talking about.

PEGS. You know damn well what I'm talking about slut, and you're not gettin away with it. I got the best lawyer in this city workin on the case and we are gonna win hands down. And not only are we gonna get our baby, but you are gonna pay us for damages through the TEETH, understand?

DEE. I'm sorry, I really think you have the wrong apartment. 
MACK. What is this?

PEGS. You the husband are ya? How's it feel to be married to a twotimin' slut who gives babies away?

MACK. Look, I'm sure this has all arisen from a misunderstanding, surely we can....

PEGS. There's no misunderstandin here. Your wife had sexshul relations with my son... on this floor... and made him do funny things [...]. (Thompson, 1987, pp. 41-42; 1989, p. 154)

Dans la version québécoise, ces répliques portent toutes les marques du même parler populaire (mises aussi en italiques) :

MACK. Ah come on! On va toute s'asseoir!!

DEE. Si vous partez pas, j'appelle la police.

MACK. Tas pas besoin d'appeler la police ma chérie, on a juste à écouter ce que la madame à dire.

DEE. Ben moi je m'en vas, vous pouvez rester si vous voulez.

MACK. Dee, envoye, relaxe.

PEGS. C"est assez le niaisage. Si toi tu penses que tu vas donner mon petit-fils ou ma petite-fille, va falloir que tu me passes sur le corps, c'est-tu clair? (Pause) M'entends-tu?

DEE. Je m'excuse, je sais vraiment pas de quoi vous parler [sic].

PEGS. Tu sais très bien de quoi je parle maudite guidoune, pis tu t'en sortiras pas comme ça. J'ai le meilleur avocat en ville qui travaille sur notre cas pis on vas gagner les doigts dans le nez. Pis non seulement on va avoir notre bébé, mais vous allez payer pour les dommages avec vos dents, vos dents, compris?

DEE. Je m'excuse, chu sûre que vous vous êtes trompés d'appartement. MACK. Qu'est-cé qui se passe?

PEGS. Cest vous le mari? Comment qu'on sent [sic] quand on est marié a une guidoune qui donne ses bébés?

MACK. Ecoutez, chu sûr que c'est un malentendu, on peut certainement...

PEGS. Y a pas de malentendu icitte. Votre femme a eu des relations sexuelles avec mon fils... sur ce plancher-là [...]. (Thompson, 1990, p. 43)

L'homogénéité linguistique appliquée au texte d'arrivée a pour conséquence d'annuler l'antagonisme de classes qui intervient pourtant dans le choix que fait Dee de ne pas remettre l'enfant à Toilane, chose que Pegs met en évidence lorsqu'elle dit : she didn't want her baby with people like us " (Thompson, 1987, p. 42; 1989, p. 154). C'est ainsi qu'elle persuadera son fils de passer à l'action : " Are you gonna let the high 
classes chew ya up and spit ya out? Are you gonna let them take your baby? "(Thompson, 1987, p. 47; 1989, p. 160). Avec tous les personnages dotés du même patois indiquant leur appartenance à la même classe sociale, comment expliquer un refus devenu injustifiable? Non que l'irrationnel soit étranger à cette pièce d'une auteure fascinée par les pulsions inconscientes de ses personnages, mais il demeure qu'une fois l'antagonisme de classes aboli les spectateurs n'ont d'autre choix que de transporter le conflit sur le seul terrain du couple et du rapport de force entre les sexes. Ce déplacement joue en faveur du père puisque Toilane, ainsi débarrassé de la tare que constitue son appartenance à une classe sociale inférieure, devient la victime innocente d'une guerre des sexes. Devant l'être socialement démuni, qui affichait auparavant les marques menaçantes de son aliénation, n'apparaî́t plus qu'un homme méprisé, un père rejeté avec lequel nous ne pouvons que sympathiser. Ce procédé linguistique vient renforcer le message déjà livré par le titre, adhérant ainsi à une isotopie thématique propre à la version québécoise ${ }^{8}$. L'isotopie à l'cuvre ici, et déjà active dans la stratégie titulaire, a pour effet de promouvoir les droits du père.

Ce nivellement linguistique par le bas aura sans doute influencé la réception de la pièce dans le contexte québécois. Depuis la célébre production des Belles-Soeurs de Michel Tremblay en 1968, le recours à la langue parlée québécoise comme langue de scène est une norme dominante du théâtre écrit et traduit au Québec. Toutefois, le francoquébécois a varié considérablement depuis : * Auparavant confiné à un niveau populaire, archaïsant et fortement anglicisé, il parcourt aujourd'hui tout le registre des niveaux de langue et coïncide souvent, à ses niveaux superieurs, avec le français "standard" "(Denis, 1990, p. 12). Or, le choix de s'en tenir à un joual fortement marqué comme langue de traduction, combiné au style exubérant de Thompson, n'est pas sans rappeler les premières pièces de Michel Tremblay. C'est une comparaison à laquelle les critiques québécois n'ont pu résister et qui, de toute évidence, ne pouvait que porter préjudice à la pièce de Thompson. Car, comment soutenir la comparaison à ce qu'un critique cité auparavant appelle $\alpha$ le

${ }^{8}$ Selon A. J. Greimas, une isotopie est un * faisceau de catégories sémantiques redondantes, sous-jacentes au discours considéré • (1970, p. 10), qui permettent une lecture uniforme du texte. 
génie de Tremblay w avec une auvre qui en reproduit les effets de langue mais doit, pour ce faire, être dépouillée de l'antagonisme de classes sur lequel est fondé le conflit quj la motive? Cette stratégie langagière aura en outre contribué, de concert avec le titre, au manque de profondeur psychologique et au " plat déterminisme » déplorés par d'autres critiques mentionnés plus haut. Ainsi, ce procédé de traduction aura joué un rôle décisif dans la réception critique de la pièce.

Fait intéressant à souligner, la critique Micheline Letourneur conçoit que l'effacement des différences de classes dans cette production soit attribuable avant tout à la mise en scène. Après avoir réprouvé le choix du décor, faisant du logement de Dee u un séjour sale et sans attrait, aussi loin que possible de l'intérieur confortable de personnes éduquées (Mack est libraire) " (Letoumeur, 1991, p. 172), la critique déplore un " manque d'acuité, chez Claude Poissant, en ce qui concerne l'appréhension des appartenances sociales " (Letoumeur, 1991, p. 172), observé déjà lors d'une production antérieure. Ce qui nous intéresse dans cette remarque, c'est qu'elle associe une caractéristique fondamentale du texte traduit à une disposition, consciente ou non, du metteur en scène. Lorsqu'on sait que la vision de ce dernier détermine tout l'ensemble du spectacle, on est en droit de se demander dans quelle mesure le nivellement linguistique de la traduction relève d'une conception globale à laquelle le texte a dâ correspondre.

En situant le texte dans le champ de la traduction théâtrale, on peut aussi relier ce choix linguistique à l'état du champ au moment où cette traduction voit le jour. À la fin des années quatre-vingt, on assiste à une mise à l'écart du modèle de l'adaptation thêâtrale, qui avait dominé la traduction québécoise du texte anglo-canadien depuis $1969^{9}$. Le recours à l'adaptation fait alors l'objet de vives critiques de la part des praticiens de la traduction théatrale et on questionne la pertinence d'une transposition systématique de l'action de la pièce empruntée en contexte québécois ${ }^{10}$. Rompant avec le modèle auparavant en vigueur, Je suis à toi

"A ce sujet, voir Ladouceur, « Du spéculaire au spectaculaire : le théâtre anglocanadien traduit au Québec au début des années 90 \% (1997a).

${ }^{10}$ Voir à ce sujet l'article de Jean-Luc Denis intitulé * Traduire le théâtre en contexte québécois : essai de caractérisation d'une pratique * (1990). 
innove en ce sens que la traduction conserve les référents onomastiques et toponymiques du texte anglo-canadien, qui affiche ainsi son origine. L'emploi exclusif du joual se présente alors comme une stratégie compensatoire d'appropriation du texte emprunté. Puisque l'adaptation et la transposition qu'elle autorisait sont à éviter, on se rabat sur l'emploi d'une langue locale fortement marquée afin d'approprier le texte au contexte récepteur.

L'analyse de cette traduction révèle aussi certains déplacements qui semblent participer d'une autre isotopie, celle de l'amour inconditionnel pour l'enfant qui va naître, sentiment prôné déjà dans la métaphore du programme nous exhortant à tendre les bras vers l'enfant qui implore. C'est dans un monologue de Dee que cette thématique se fait surtout sentir. Dans la version publiée en 1989, Dee est déchirée par des désirs contradictoires au moment de subir un avortement :

DEE. Is that you? Are you ... speaking ... to me? I can hear you breathing, speaking. STOP, PLEASE! STOP SPEAKNNG TO ME NOOOO! I don't want to hear you STOP. STOP talking to me - you're breathing, in my ears, stop. Please, no! I DON'T want to KNOW you, NO, PLEASE, I WANT TO GET RID OF YOU I - don't. Don't. DON'T make those ... (she sees something that touches her - such as a baby's smile, a small hand, etc.) don't-no, no no no OKAY! OKAY OKAY OKAY YOU ARE! You are! You are!! YOU ARE!!! (Thompson, 1989, p. 143)

Ce passage a été révisé par Thompson pour la publication puisque dans la version de 1987 on lisait :

DEE. (looks at the audience) Is that you?? Are you ..speaking...to me? I can hear you. I can hear you - I hear...your voice, are you.. I can hear your voice - speaking. It's you!!! It isss.....you, you are talking to me. You're talking to me, yes I can hear, I can feel these, yes, I can hear you breathing, breathing, your voice, your step - the way you will step on the stairs, a small intake after you laugh, you laugh, the way your mouth..., when you sleep; curves down; the swell...inside you when you see a tree, a beautiful tree one day, a shudder of cold, your hand being squeezed, your eyes looking at me! $\mathrm{Oh}$ ! you.....,are ...you are......you.....ARE!!!!!!!! (Thompson, 1987, p. 28) 
Dans la traduction québécoise, on s'est inspiré de la version manuscrite de 1987, dans laquelle est proposé un discours où l'amour maternel demeure incontesté :

DEE. (regardant le public) Je t'entends. Je t'entends.. J'entends...ta voix, es-tu...Je peux entendre ta voix qui parle. C'est toi!!! Oui!!!! c'est toi, tu me parles. Tu me parles oui, j'entends, je te sens respirer, respirer, ta voix, la façon dont tu vas monter les marches...une petite entrée d'air apres ton rire, comment ta bouche va....quand tu dors; la petite boule...en-dedans de toi quand tu vois un arbre. Un petit frisson, ta main qu'on serre, tes yeux qui me regardent! (Thompson, 1990, p. 30)

Qu'on se soit inspiré de la version manuscrite antérieure à la publication suggère deux possibilités : soit qu'on ignorait que la pièce avait été publiée dans l'anthologie The Other Side of the Dark, célèbre pourtant puisqu'elle remportait le Prix du Gouverneur général en 1989, donc l'année précédant la traduction et sa production en novembre 1990, soit qu'on a écarté cette version publiée et plus récente au profit d'un discours où l'amour maternel ne souffre d'aucune incertitude et où l'avortement est impensable.

La fonction matemelle est d'ailleurs le thème qui fait l'objet des substitutions les plus notables dans la traduction québécoise. Par exemple, dès les premières lignes, la traduction s'écarte des versions originales de 1987 et 1989 pour insister sur la relation entre Dee et sa mère. Plutôt que de commencer par la réplique : x Mum! Mum! I'm home!... "(Thompson, 1987 , p. 1; 1989, p. 110) dite par Toilane, qui est aussi le seul personnage de cette première scène, la version québécoise lance la pièce avec un cri de Dee : « Moman! " (Thompson, 1990, p. 1), suivi des répliques de Toilane. Cet ajout impose une lecture axée d'abord sur la relation de Dee avec sa mère et crée un sous-texte qui prendra par la suite une ampleur inattendue.

En effet, plus loin dans la pièce, on apprend que Dee souffre d'avoir été cruellement rejetée par sa mère au profit de sa soeur Mercy, ce qui serait la cause d'affreux cauchemars. Lorsqu'elle avoue à Mercy avoir eu un rêve érotique horrible, celle-ci lui répond : * How horrible could it be, were you devouring Mummy's brains and spitting out her teeth.... * (Thompson, 1989, p. 140; 1987, p. 26). Cette hyperbole reliée à l'image de la mère exprime un degré potentiel d'horreur dans le rêve et, en ce 
sens, demeure totalement hypothétique. Or, ce n'est pas le cas dans la version québécoise où Mercy réplique : *Comme quand tu disais que to boufferais le cerveau de moman pis qu'tu recracherais ses dents? " (Thompson, 1990, p. 28). L'effet dramatique intense ménagé par cette reformulation n'est pas sans conséquence sur le discours vêhiculé ici. L'allusion à une haine féroce de la part de Dee envers sa mère, une haine qu'elle aurait avouée en termes des plus violents et dont Mercy aurait encore le souvenir, ne peut que discréditer davantage son rapport à la fonction maternelle, altérant encore à son détriment la dynamique parentale qui l'oppose à Toilane.

Une suppression ultérieure a d'ailleurs pour résultat de passer sous silence certains traits révélateurs de la relation entre Dee et sa mère et du rapport à la maternité qui en découle. Dans l'extrait suivant, emprunté aux deux versions originales, la conversation au sujet du rêve de Dee se poursuit. La partie supprimée par la traduction est en italiques :

DEE. I am afraid. I am afraid that the dreams will seep into the day. That I'll do things - that I'll...

MERCY. Is that why you broke up with Mack. Dee? Is it?

DEE. I don't know. He's the only person I ever wanted. I don't -

MERCY. Well, sounds to me like you did the right thing.

DEE. I did?

MERCY. Well yes. I mean, a man would bring this thing forth, wouldn't he? Or a baby. Dee, you mustn't have a baby.

DEE. Why?

MERCY. Who knows what might happen. Who knows what you could do. You could do horrible things. Mum knew that about you - Dee? Knife old ladies in the head. Screw old winos in the park. When people let their animal out they go to the top of tall buildings and shoot forty people.

DEE. Oh God.

MERCY. I know you Dee, I'm your sister. Mum knew you. I know what you could do. No, you don't want Mackie. I'm here now. I'll take care of you. I know you.... (Thompson, 1987, p. 26; 1989, p. 141)

Pourquoi faire fi de ces passages fort révélateurs de la dynamique psychologique dont les personnages sont investis? Pourquoi supprimer la peur des comportements pathologiques que la maternité pourrait entraîner chez Dee? Qui plus est, pourquoi éliminer l'allusion à la mère comme source principale de cette perception de soi? En fait, pourquoi ne pas 
avoir simplement supprimé tout le passage concernant la possible maternité et ses horribles conséquences? L'injonction de Mercy prend tout son intérêt du fait qu'on apprend dans la scène suivante que Dee est effectivement enceinte. On l'a donc conservée pour l'effet dramatique qu'elle instaure. Mais on aura pressenti que la peur de "l'animal " intérieur, thème crucial de la pièce, et l'allusion aux actes horribles que la maternité pourrait engendrer chez Dee auraient pu agir de façon à légitimer la décision de ne pas avoir l'enfant. Peut-être a-t-on aussi préféré ne pas insister sur la problématique particulière de la relation mère-fille? Quoi qu'il en soit, on se trouve ici devant des choix qui relèvent d'une éthique ou d'une esthétique subjective dont les motifs demeurent partiellement obscurs.

Autre fait très intéressant, la version manuscrite révèle que bon nombre de ces changements ont été effectués après la rédaction d'une première version par le traducteur, dans laquelle certains passages avaient été laissés en blanc. On a par la suite inscrit à la main les solutions retenues, dont la première réplique ajoutée à la version traduite et celle où Mercy décrit le rêve de sa sœur. Comment a-t-on résolu le problème, rarement linguistique, que posait la traduction de ces passages? Les solutions retenues sont-elles issues d'une consultation avec le metteur en scène ou avec d'autres membres de l'équipe de production? Sont-elles le produit d'une lecture effectuée par les comédiens ou se sont-elles dessinées au cours des répétitions? Autant de questions qui laissent supposer l'action d'agents intermédiaires dans l'élaboration du produit final.

Selon Robert Vézina, il n'y aurait pas lieu de s'étonner de tels glissements thématiques puisqu'il commente ainsi l'approche adoptée pour traduire la pièce :

You translate a play for Quebec very differently than you would for a production in France. Not just the differences in language, but the things you punch up in the text too. In some small instances it's not just the words, it might even be the themes. (Charlebois, 1991, p. 20)

À la question de savoir comment Judith Thompson réagit à cette façon de traduire son texte, voici le commentaire qu'elle faisait au journaliste 
Gaëtan Charlebois après avoir assisté à une représentation de Je suis à toi à Montréal en 1990 : "What's Vézina like, and is he doing a good job? I don't understand French very well. It seemed right, the way the audience responded "(Charlebois, 1991, p. 20). Vézina ajoute dans le même article : $~ \&$ heard that she was pleased with the translation of $I$ Am Yours because when she came to see it, people laughed at the right places" (Charlebois, 1991, p. 20). Qu'il soit le plus souvent impossible pour l'auteur, d'une part, et pour la communauté linguistique visée, d'autre part, d'évaluer le travail effectué sur le texte original par la traduction contribue à en maintenir l'invisibilité.

Enfin, un commentaire dans lequel Claude Poissant exprime sa conception du traduire nous éclaire sur la dynamique à l'œuvre dans la production du texte en traduction. L'adaptateur de la pièce de Kobo Abe Les amis, pour laquelle il a remporté le prix de la meilleure adaptation pour la scène décerné par l'Association québécoise des critiques de théâtre en 1990, prenait position dans un numéro des Cahiers de thédtre Jeu consacré à la traduction théâtrale. Dans un texte introduit par la question " Est-il préférable d'adapter une cuvre dramatique étrangère à la réalité immédiate du public visé? », Poissant faisait appel à son expérience de metteur en scène lors de l'événement Pièces en transit organisé par le Centre des auteurs dramatiques à Limoges en 1988, où il a dirigé la lecture de la pièce de Madeleine Lalk Voyageurs. Il concluait ainsi : « Oui, adapter c'est trahir, et tant mieux. Trahir pour la bonne cause. Pour faire entendre ce qu'on a envie de dire. C'est un travail de mise en scène avant tout " (Poissant, 1990. p 71). Bien qu'il soit ici question d'adaptation et non de traduction, on retiendra de ce commentaire qu'il subordonne le texte emprunté à ce que le metteur en scène, " avant tout ", " a envie de dire x. C'est une attitude qui n'est sans doute pas étrangère à certains choix de traduction effectués pour la pièce de Judith Thompson.

\section{Conclusion}

Comme j'ai tenté de le démontrer, la sélection de la pièce à traduire, dont le propos s'insérait dans le cadre d'un débat qui avait récemment bouleversé et divisé la société d'accueil, ainsi que les stratégies appliquées à sa traduction ont eu pour effet de promouvoir certaines valeurs au sein du contexte social récepteur. Bien qu'elles nourrissent un débat de sociêté, ces valeurs sont, à l'origine, prises en charge par des sujets qui les 
expriment et s'en font les porteurs, critiques ou défenseurs, selon les intérêts dont elles sont l'enjeu.

Dans le cas étudié ici, on peut observer l'effet d'une subjectivité aux prises avec les représentations du rapport à la mère et à la maternité véhiculées par le texte. Cette relation conflictuelle entre la position traductive du traducteur et les exigences spécifiques de l'œuvre à traduire se manifeste par la suppression des énoncés révélant les effets pathologiques que pourrait entrainer la grossesse et l'allusion à la mère comme source d'énonciation de ce comportement hypothétique. $\dot{A}$ un autre niveau, certains choix de traduction semblent plutôt procéder d'une axiologie de groupe occupée à promouvoir les droits du père et une acceptation inconditionnelle de l'enfant à venir. Ces thématiques sont mises en relief par l'exhortation implicite contenue dans le programme, l'évident parti pris dont témoigne le titre, luniformité imposée au langage et une subtile invalidation du rapport à la fonction matemelle représenté par Dee. Le nivellement linguistique du texte pourrait en outre relever de l'état du champ de la traduction théâtrale québécoise au moment où il est produit. Enfin, divers remaniements apportés à la pièce ont pour effet d'exprimer une position idéologique au sein du discours social récepteur.

Les conclusions proposées par cette étude soulèvent implicitement l'épineuse question de la responsabilité du traducteur envers un texte avec lequel il entretient des rapports antagonistes. La question, trop vaste pour être débattue dans le cadre de cet article, invite toutefois à la réflexion sur l'ingérence, consciente et inconsciente, du sujet traduisant dans le texte-cible et l'invisibilité dont elle jouit. Cette invisibilité constitue un lieu privilégié où les subjectivités à l'œuvre, que ce soit au niveau de la traduction, de la mise en scène, de la production ou de la publication, peuvent moduler la portée idéologique du texte emprunté, lequel est transmis à un public qui, le plus souvent, n'a aucune idée des enjeux dont l'emprunt fait l'objet.

Université Laval

\section{Références}

ANGENOT, Marc (1979). Glossaire pratique de la critique littéraire contemporaine. Montréal, Hurtubise. 
BEAUNOYER, Jean (1990). * Mince! Si la vie ne veut plus rien dire ". $L a$ Presse [Montréal], 17 nov., p. D8.

BEMROSE, John (1987). * Love Among the Ruins ». Maclean's, 30 nov., p. 65 .

BERMAN, Antoine (1995). Pour une critique des traductions: John Donne. Paris, Gallimard.

BOULANGER, Luc (1990). * Illusion comique ". Voir [Montréal], 22-28 nov., p. 28.

BOURDIEU, Pierre (1980). Questions de sociologie. Paris, Les Éditions de Minuit.

BRISSET, Annie (1990). Sociocritique de la traduction. Thedtre et altérité au Québec (1968-1988). Longueuil, Le Préambule.

CHARLEBOIS, Gaětan (1990), * Va-t-en *. Mirror [Montréal], 29 nov.-6 déc., p. 25.

- (1991). * Breaking Boundaries w. Mirror [Montréal], 26 sept.-3 oct., pp. 20-21.

CONLOGUE, Ray (1993). « Quebec's Surprising New Wave ». The Globe and Mail [Toronto], 26 jan., p. A12.

CREW, Robert (1987). * Thompson Play True to Form ». The Toronto Star, 18 nov., p. Bl.

DENIS, Jean-Luc (1990). * Traduire le thếtre en contexte québécois : essai de caractérisation d'une pratique n. Cahiers de théatre Jeu, 56, pp. 917.

DONNELLY, Pat (1990). * Je Suis à Toi is tale of gripping black humor. La Licorne adapts Thompson play ». The Gazette [Montréal], 26 sept., p. D13. 
GAREBIAN, Keith (1988). $\propto$ I Am Yours by Judith Thompson $n$. Queen's Quarterly, 95, 2, pp. 232-234.

GOUANVIC, Jean-Marc (1997), Pour une sociologie de la traduction : le cas de la littérature américaine traduite en France après la Seconde Guerre mondiale (1945-1960) n. Translation as Intercultural Communication. Ed. Mary Snell-Hornby, Zuzana Jettmarova et Klaus Kaindl. Amsterdam, Benjamins, pp. 33-44.

- (1994). * La traduction et le devenir social : le cas de l'irruption de la science-fiction américaine en France après la Seconde Guerre mondiale ". $T T R, 7,1$, pp. 117-152.

GREIMAS, A. J. (1970). Du Sens. Paris, Seuil.

KOWZAN, Tadeusz (1975). Littérature et spectacle. La Haye et Paris, Mouton.

LADOUCEUR, Louise (1997a). * Du spéculaire au spectaculaire : le theâtre anglo-canadien traduit au Québec au début des années 90 \%. Nouveaux regards sur le théâtre québécois. Ed. Betty Bednarski et Irene Oore. Montréal et Halifax, XYZ Éditeur/Dalhousie French Studies, pp. 185-194.

- (1997b). * Separate Stages : la traduction du thétre dans le contexte Canada/Québec n. These de doctorat, The University of British Columbia.

LEDUC, Jean-Denis et Daniel SIMARD (1989). Programme de Je suis a toi. Montréal, Thếtre de la Manufacture.

LETOURNEUR, Micheline (1991). * Je suis à toi de Judith Thompson *. Cahiers de théâtre Jeu, 58, pp. 170-72.

LOTBINIĖRE-HARWOOD, Susanne (1991). Re-Belle et Infidèle. La traduction comme pratique de réécriture au féminin/The Body Bilingual. Translation as a Rewriting in the Feminine. Montréal et Toronto, Les Éditions du remue-ménage et Women's Press. 
NUNN, Robert (1991). « Canada Incognita : Has Quebec Theatre Discovered English Canadian Plays? $"$ Theatrum, 24, pp. 14-19.

POISSANT, Claude (1990) * Adapter : le choix de trahir *. Cahiers de théâtre Jeu, 56, pp. 69-72.

THOMPSON, Judith. (1987). I Am Yours. Manuscrit inédit, Toronto, Great North Artists Management.

- (1989). "I Am Yours ". The Other Side of the Dark. Toronto, Coach House, pp. 117-176.

- (1990). Je suis à toi. Traduction de Robert Vézina, manuscrit inédit, Montréal, École nationale de thêatre.

TOURY, Gideon (1995). Descriptive Translation Studies and Beyond. Amsterdam, Benjamin.

- (1980). In Search of a Theory of Translation. Tel Aviv, The Porter Institute for Poetics and Semiotics.

UBERSFELD, Anne (1978). Lire le théâtre. Paris, Éditions Sociales.

VENUTI, Lawrence (1995). The Translator's Invisibility. A History of Translation, New York, Routledge.

VON FLOTOW, Luise (1995), " Translating the Women of the Eighties : Eroticism, Anger, Ethnicity ". Culture in Transit : Translating the Literature of Quebec. Sherry Simon, dir. Montréal, Véhicule Press.

RÉSUMÉ : Le Sujet en question : I Am Yours de Judith Thompson, version québécoise - Si certains choix de traduction sont orientés par les normes collectives en vigueur dans le contexte récepteur, il en est d'autres qui relèvent de l'espace plus restreint du champ dans lequel s'inscrivent l'activité de traduction et l'agent qui la pratique. D'autres encore procèdent des subjectivités participant à la production de l'œuvre en traduction. Cet article traite des relations qu'entretient le sujet traduisant avec ces nombreuses contraintes dans Je suis à toi, version 
québécoise de la pièce I Am Yours de l'auteure canadienne-anglaise Judith Thompson.

\begin{abstract}
The Subject in Question : A Quebecois Version of Judith Thompson's I Am Yours - If some translative choices are oriented by the collective norms active in the receptive context, others depend on constraints related to the narrower space of the field to which the translation and the translator belong. Further choices originate in the subjectivity of the individuals involved in producing the translation. This article looks at the translator as a subject confronted with such constraints in Je suis à toi, a Quebecois version of Canadian playwright Judith Thompson's I Am Yours.
\end{abstract}

Louise Ladouceur : Centre de recherche en littérature québécoise, Faculté des lettres, Pavillon Charles-De-Koninck, Université Laval, Sainte-Foy (Québec), G1K 7 P4.

Courriel : louise.ladouceur@creliq.ulaval.ca 